\title{
Magnetic Scaling Laws for the Atmospheres of Hot Giant Exoplanets
}

\author{
Kristen Menou ${ }^{1}$
}

\begin{abstract}
We present scaling laws for advection, radiation, magnetic drag and ohmic dissipation in the atmospheres of hot giant exoplanets. In the limit of weak thermal ionization, ohmic dissipation increases with the planetary equilibrium temperature $\left(T_{\text {eq }} \gtrsim 1000 \mathrm{~K}\right)$ faster than the insolation power does, eventually reaching values $\gtrsim 1 \%$ of the insolation power, which may be sufficient to inflate the radii of hot Jupiters. At higher $T_{\text {eq }}$ values still, magnetic drag rapidly brakes the atmospheric winds, which reduces the associated ohmic dissipation power. For example, for a planetary field strength $B=10 \mathrm{G}$, the fiducial scaling laws indicate that ohmic dissipation exceeds $1 \%$ of the insolation power over the equilibrium temperature range $T_{\mathrm{eq}} \sim 1300-2000 \mathrm{~K}$, with a peak contribution at $T_{\text {eq }} \sim 1600 \mathrm{~K}$. Evidence for magnetically dragged winds at the planetary thermal photosphere could emerge in the form of reduced longitudinal offsets for the dayside infrared hotspot. This suggests the possibility of an anticorrelation between the amount of hotspot offset and the degree of radius inflation, linking the atmospheric and interior properties of hot giant exoplanets in an observationally testable way. While providing a useful framework to explore the magnetic scenario, the scaling laws also reveal strong parameter dependencies, in particular with respect to the unknown planetary magnetic field strength.
\end{abstract}

\section{Introduction}

Hot giant exoplanets, including hot Jupiters, are among the best characterized exoplanets. Secondary eclipses, transmission spectroscopy and orbital phase curves in particular have provided constraints on the atmospheric properties of several transiting members of this class (see Charbonneau 2009; Winn 2010 for reviews). Increasingly detailed radiative and circulation models for the atmospheres of these exoplanets have also been developed to help interpret the rich emerging phenomenology (see Burrows \& Orton 2010; Showman et

\footnotetext{
${ }^{1}$ Department of Astronomy, Columbia University, 550 West 120th Street, New York, NY 10027
} 
al. 2010 for reviews). Finally, evolutionary models have been systematically used to infer the bulk interior properties of hot giant exoplanets with precisely measured transit radii (see Baraffe et al. 2010 for a review).

It was recently proposed that magnetic effects could have important consequences for the atmospheric and bulk interior properties of hot giant exoplanets. Magnetic drag and ohmic dissipation, which result from kinematic induction by thermally-driven winds in the weakly-ionized atmospheres of hot giant exoplanets 1 will indeed brake atmospheric winds (Perna et al. 2010a; Rauscher \& Menou 2011) and deposit extra heat in the deep atmosphere or the interior adiabat (Batygin \& Stevenson 2010; Perna et al. 2010b), thus affecting some of the observable properties of these exoplanets.

Here, we establish simple scaling laws for the strength of magnetic drag and ohmic dissipation in the atmospheres of hot giant exoplanets, as a function of their radiative equilibrium temperature. These scaling laws allow us to study the nature of the transition into the magnetized regime, as the radiative equilibrium temperature is raised above $\sim 1000 \mathrm{~K}$, and to explore how the limit of strong coupling with the planetary magnetic is eventually reached, at higher temperatures. The remainder of this paper is organized as follows. In $\S 2$, we recall the basic mechanism of magnetic induction in the atmosphere of a hot giant exoplanet. In $\S 3$, we establish scaling laws for the strength of magnetic drag and ohmic dissipation as a function of the planetary radiative equilibrium temperature. The main results emerging from these scaling laws are described in $\S 4$. In $\S 5$, we discuss various parameter dependencies, the strength of induced magnetic fields, connections to previous work on this topic and a few plausible observational signatures of magnetic effects in the population of hot giant exoplanets. We conclude in $\S 6$.

\section{Basic Mechanism}

Day-side insolation on a hot Jupiter generates fast atmospheric winds (see, e.g., reviews by Showman et al. 2008; 2010). As the weakly-ionized gas flows across the planetary magnetic field, which is presumably anchored to the planet's bulk rotation via deep-seated electric currents in the convective interior, an additional magnetic field is induced in the atmosphere. In steady-state, this magnetic induction is balanced by resistive diffusion and associated ohmic dissipation in the resistive atmosphere.

\footnotetext{
${ }^{1}$ We are excluding from our analysis giant exoplanets which are hot because of their young age. While magnetic effects may also be important for them, our focus is on older planets with atmospheric temperatures and winds determined by strong external irradiation, as exemplified by hot Jupiters.
} 
Let us consider a spherical coordinate system $(r, \theta, \phi)$ that is rotating with the planet. Let us further assume that the planetary magnetic field takes the form of an aligned dipole (of surface strength, $B_{\text {dip }}$ ) and that the dominant atmospheric flow is zonal (azimuthal) in nature: $V_{\phi} \gg V_{r}, V_{\theta}$. To leading order, the induced current $J$ satisfies the steady-state resistive induction equation

$$
\frac{\partial \mathbf{B}}{\partial t}=\nabla \times\left(\mathbf{V}_{\phi} \times \mathbf{B}_{\text {dip }}\right)-\nabla \times\left(\frac{4 \pi \eta}{c} \mathbf{J}\right)=0,
$$

where $\eta$ is the local resistivity of the weakly-ionized atmospheric gas. Note the implicit assumption that the deep-seated electric currents generating the dipolar planetary field are located well below the atmospheric region of interest, so that they can be omitted from the above induction equation (Liu et al. 2008; Batygin \& Stevenson 2010; Perna et al. 2010a,b).

For a purely zonal flow and a strictly aligned dipole, a purely toroidal field $\left(B_{\phi}\right)$ is induced, which is sustained by purely poloidal currents: $\mathbf{J}=(c / 4 \pi) \nabla \times \mathbf{B}_{\phi}$ (see, e.g., Liu et al. 2008). Magnetic induction does not operate at the rotational equator for an aligned dipole because vertical speeds are negligibly small and meridional wind speeds do not contribute. The aligned dipole assumption is likely to remain qualitatively correct even for moderate levels of misalignment.

Focusing on the dominant, zonal component of the atmospheric flow, a representative horizontal momentum balance for the steady flow in the azimuthal $\left(\mathbf{e}_{\phi}\right)$ direction may be written as

$$
\left(\mathbf{V}_{\phi} \nabla \mathbf{V}_{\phi}\right) . \mathbf{e}_{\phi}=\left(-\frac{1}{\rho} \nabla P-\frac{1}{\rho c} \mathbf{J} \times \mathbf{B}_{\text {dip }}\right) . \mathbf{e}_{\phi}
$$

where the zonal acceleration (LHS) resulting from the day-night pressure gradient (first term on the RHS) is reduced by the bulk Lorentz force due to magnetic drag (second term on the RHS; e.g., Zhu et al. 2005).

Our main goal with this work is to study how this momentum balance changes with the atmospheric resistivity, which depends itself strongly on the planet's radiative equilibrium temperature via thermal ionization balance. Considering the above induction and momentum equations together, one anticipates the following behavior. At low enough atmospheric temperatures (high resistivity $\eta$ ), magnetic drag $(\propto J)$ will not significantly reduce the zonal wind speed, $V_{\phi}$ (in Eq. [2]). At fixed $V_{\phi}$ and $B_{\text {dip }}$ values, the induced current $J$ will thus increase in inverse proportion to the resistivity $\eta$ (in Eq. 1]). At large enough temperatures (low resistivity $\eta$ ), however, the winds will be strongly dragged and it is no longer obvious how wind speeds and induced currents should scale with resistivity in Eq. (1). It is thus of great interest to establish the range of atmospheric temperatures over which such a transition occurs and to clarify what happens in the limit of high conductivity (high temperatures). 


\section{Magnetic Scaling Laws}

Our goal in establishing magnetic scaling laws for hot giant exoplanet atmospheres is not to obtain an accurate description of magnetic effects for this class of planets, as this will require detailed multi-dimensional models. Rather, our focus is on the leading-order scaling with the radiative equilibrium temperature, which sets the atmospheric resistivity. As we establish these scaling laws, we will need to invoke important simplifying assumptions.

\subsection{General Properties}

We adopt fiducial parameters for the scaling laws that represent a typical hot Jupiter, with a hydrogen-dominated, solar composition atmosphere, so that a perfect gas constant $\mathcal{R}=4.59 \times 10^{7} \mathrm{erg} / \mathrm{g} / \mathrm{K}$ and a specific heat at constant pressure $C_{p}=1.43 \times 10^{8} \mathrm{erg} / \mathrm{g} / \mathrm{K}$ are appropriate. The planetary radius is $R_{p}=10^{10} \mathrm{~cm}$, with a surface gravity $g=890 \mathrm{~cm} \mathrm{~s}^{-2}$. We explore surface magnetic field values from $B_{\text {dip }}=3 \mathrm{G}$ to $B_{\text {dip }}=30 \mathrm{G}$. Planetary radiative equilibrium temperatures ranging from $T_{\text {eq }}=1000 \mathrm{~K}$ to $2200 \mathrm{~K}$ are considered, which correspond to semi-major axes $a \simeq 0.09-0.02$ AU around a Sun-like star 2

\subsection{Model Atmospheres}

For the purpose of the present study, we focus our discussion on the most actively forced, "weather layer" region of the atmosphere, defined here as the region located between the thermal (infrared) photosphere above and the insolation (visible) photosphere below, which corresponds to a level above which $\sim 50 \%$ of the insolation flux has been absorbed (see details below). For simplicity, the vertical thermal structure of the atmosphere is approximated with the one-dimensional radiative solution discussed by Guillot (2010; see also Hansen 2008) for a zero incidence angle and a dilution factor $f=0.5$ representing a dayside average. As shown by Guillot (2010; see his Fig. 4), this solution provides reasonable temperature-pressure profiles for hot Jupiter atmospheres over a range of orbital radii, even though it clearly is a simplified approach and it neglects important three-dimensional aspects of the atmospheric structure. In what follows, we refer to this pressure-dependent temperature profile for the dayside weather layer as $T_{\text {day }}$. Note that, by construction, $T_{\text {day }} \gtrsim T_{\text {eq }}$ everywhere in this

\footnotetext{
${ }^{2}$ The radiative equilibrium temperature is defined by $T_{\text {eq }}=T_{*}\left(\frac{R_{*}}{2 D}\right)^{1 / 2}$ for zero Bond albedo, where $T_{*}$ and $R_{*}$ are the stellar effective temperature and radius, and $D$ is the planet-star orbital separation (e.g., Guillot 2010).
} 
weather layer model.

Following Guillot (2010), a single thermal absorption coefficient $\kappa_{\text {th }}=10^{-2} \mathrm{~cm}^{2} \mathrm{~g}^{-1}$ and a single visible absorption coefficient $\kappa_{\mathrm{v}}=4 \times 10^{-3} \mathrm{~cm}^{2} \mathrm{~g}^{-1}$ are adopted. For these values, the thermal and visible photospheres (defined by $\tau=2 / 3$ ) are located, respectively, at $P_{\text {th }} \simeq 60$ mbar and $P_{\mathrm{v}} \simeq 150$ mbar (above which $\sim 50 \%$ of the stellar flux has been absorbed). The number of pressure scale heights over which the weather layer extends vertically in our models is thus modest, $\Delta \ln P=\ln P_{\mathrm{v}}-\ln P_{\mathrm{th}} \simeq 0.9$. An internal heat flux corresponding to $T_{\text {int }}=150 \mathrm{~K}$ is adopted for the thermal structure calculation, but the exact value has little effect on the atmospheric properties of the weather layer of interest here.

Guillot (2010) has also shown that these values for the grey absorption coefficients are adequate to reproduce the global radiative equilibrium balance of the prototypical hot Jupiter HD209458b, with $T_{\text {eq }} \simeq 1420 \mathrm{~K}$. The fixed values of the absorption coefficients adopted in our scaling laws, despite the large range of $T_{\text {eq }}=1000-2200 \mathrm{~K}$ considered, is arguably one of the strongest simplifying assumptions we make in our models. We comment on the effects of variations in opacities and other model parameters in $\$ 5.1$.

\subsection{Ionization Balance}

Thermal ionization, rather than photoionization, is expected to be dominant over the range of atmospheric pressures $(P \gtrsim 50$ mbar) of interest for the scaling laws (see, e.g., Perna et al. 2010a; Batygin \& Stevenson 2010). Rather than using the same approximate solution to the Saha equation as in Perna et al. (2010a,b), which is only justified at low enough temperatures $(T \lesssim 1800 \mathrm{~K})$, we use here a more general but still simplified solution to the Saha equation (e.g., Sato 1991). In the weakly-ionized regime, when only single ionization has to be considered, the ionization fraction $x_{e}(\ll 1)$ satisfies the equations

$$
\begin{aligned}
x_{e} & =\frac{n_{e}}{n_{n}}=\sum_{j} \frac{n_{j}}{n} x_{j} \\
\frac{x_{j}^{2}}{1-x_{j}^{2}} & \simeq \frac{1}{n_{j} k T}\left(\frac{2 \pi m_{e}}{h^{2}}\right)^{3 / 2}(k T)^{5 / 2} \exp \left(-I_{j} / k T\right),
\end{aligned}
$$

where $n_{e}$ and $n_{n}$ are the number densities of electrons and neutrals, respectively (in $\left.\mathrm{cm}^{-3}\right), n_{j}$ is the number density of element $j, I_{j}$ is the corresponding first ionization potential, $T$ is the temperature, $n$ is the total number density of the gas and the other symbols have their usual meaning. Solar composition for the first 28 elements of the periodic table ( $\mathrm{H}$ to 
$\mathrm{Ni}$ ) is assumed for the ionization calculations. In the limit $x_{j} \ll 1$, which ceases to be valid for some elements at $T \gtrsim 2000 \mathrm{~K}$ for the densities of interest here, the above expression essentially reduces to that used by Laughlin et al. (2011). Based on this solution for the ionization balance, the local electric resistivity of the atmospheric gas is evaluated everywhere in the weather layer as $\eta=230 \sqrt{T} / x_{e} \mathrm{~cm}^{2} \mathrm{~s}^{-1}$ (see Perna et al. 2010a for details).

The ionization solutions obtained with Eq. (44) are still approximate, however, since all the degeneracy factors in the Saha equation have been arbitrarily set to unity. This crude simplification is justified by the fact that the specific atmospheric composition of any given hot Jupiter is a priori unknown and could easily deviate from solar in a non-trivial way. In addition, from the point of view of the scaling laws, inaccuracies in the degeneracy factors are dwarfed by the exponential dependence with temperature appearing in Eq. (4), given the wide range of temperatures considered. Indeed, we verified that factor several changes in the degeneracy factors entering the Saha equation have little practical impact on our scaling law results.

\subsection{Forcing, Momentum Balance and Dragged Wind Speeds}

Showman et al. (2010) describe how to obtain a simple, order-of-magnitude scaling for the zonal wind speed in a hot Jupiter atmosphere, in the absence of magnetic drag, based

on steady non-linear balance between the zonal acceleration term and the pressure gradient term in the horizontal momentum equation. The same dimensional analysis of Eq. (2), taking the limit $B_{d i p}=0$, leads to the same scaling

$$
V_{\phi}=\sqrt{\mathcal{R} \Delta T_{\text {horiz }} \Delta \ln P},
$$

where $\Delta T_{\text {horiz }}$ is the typical day-night temperature differential along the equator, $\mathcal{R}$ is the perfect gas constant and $\Delta \ln P$ measures the number of vertical pressure scale heights in the weather layer over which this horizontal temperature differential applies.

In the presence of magnetic drag, a similar dimensional analysis of Eq. (2) leads to the balance

$$
\frac{V_{\phi}^{2}}{R_{p}}=\frac{\mathcal{R} \Delta T_{\text {horiz }} \Delta \ln P}{R_{p}}-\frac{V_{\phi} B^{2}}{4 \pi \rho \eta},
$$

where the bulk Lorentz force has been evaluated via dimensional analysis of the steady-state resistive induction equation (Eq. [1) for the latitudinal current: $J_{\theta} \sim c \mathrm{~V}_{\phi} B /(4 \pi \eta)$ (see, e.g., Perna et al. 2010a). We obtain an order-of-magnitude estimate of the zonal wind speed in the presence of magnetic drag by solving for the positive root of this quadratic equation for $V_{\phi}$. 
An additional complication with the above estimate is that the day-night temperature differential itself, $\Delta T_{\text {horiz }}$, depends on the zonal wind speed $V_{\phi}$ via the efficiency of heat advection from the dayside to the nightside. This results in a non-linear coupling between advection and radiation processes in the atmospheric flow. While Showman \& Guillot (2002) proposed to capture this dependence with an exponential relation between $\Delta T_{\text {horiz }}$ and the ratio of the atmospheric advective and radiative timescales, we adopt a power-law dependence here for simplicity, keeping the index as a free parameter. The horizontal temperature differential between the day and the night sides of a tidally-locked, hot giant exoplanet is thus estimated as

$$
\Delta T_{\text {horiz }}=\min \left[\frac{T_{\text {day }}}{2}, \frac{T_{\text {day }}}{2}\left(\frac{\tau_{\text {adv }}}{\tau_{\text {rad }}}\right)^{n}\right],
$$

so that when $\tau_{\text {adv }} / \tau_{\text {rad }}>1$, the full differential $T_{\text {day }} / 2$ is applied, while if $\tau_{\text {adv }} / \tau_{\text {rad }}<1$, advection reduces the effective temperature differential between day and night in proportion to the values of these two timescales. A default value $n=1$ is adopted for the power law index but other values are explored below.

The advective timescale is evaluated as

$$
\tau_{\mathrm{adv}}=\frac{R_{p}}{V_{\phi}}
$$

while the radiative timescale is evaluated as

$$
\tau_{\mathrm{rad}}=\frac{C_{p} P}{g \sigma T_{\mathrm{day}}^{3}}
$$

where $g$ is the gravitational acceleration, $P$ and $T_{\text {day }}$ are the pressure and dayside temperature at the location of interest in the weather layer and $\sigma$ is the Stefan-Boltzmann constant (e.g., Goody \& Yung 1989).

Equations (5) and (6) for our two unknowns, $V_{\phi}$ and $\Delta T_{\text {horiz }}$, are solved iteratively. As it turns out, for the majority of the parameter space of interest here, atmospheres are strongly radiative so that the precise form of the scaling with $\tau_{\text {adv }} / \tau_{\text {rad }}$ or $n$ in Eq. (6) has little consequences for our main conclusions.

The ohmic power dissipated per unit volume in relation to the magnetic drag is evaluated as

$$
Q_{\mathrm{ohm}}=\frac{4 \pi \eta J^{2}}{c^{2}}=\frac{V_{\phi}^{2} B^{2}}{4 \pi \eta} .
$$


Integrating vertically, and then horizontally over the volume of the weather layer, this yields an estimate of the total ohmic power dissipated as a result of induction in the resistive atmosphere,

$$
P_{\mathrm{ohm}}=4 \pi R_{p}^{2} \int_{\Delta \ln P} Q_{\mathrm{ohm}} \times H_{p} d \ln P
$$

where the local pressure scale height is evaluated as $H_{p}=\mathcal{R} T_{\text {day }} / g$. It is worth emphasizing that this estimate of the ohmic power is not expected to be accurate at better than an order of magnitude level, given that it overlooks important issues related to the geometry and the spatial distribution of electrical currents within and outside the weather layer (see, e.g., Liu et al. 2008; Batygin \& Stevenson 2010; Perna et al. 2010b).

\section{Results}

Figure 1 shows our estimate for the zonal wind speed, $V_{\phi}$ at the thermal photosphere $\left(P_{\mathrm{th}} \simeq 60 \mathrm{mbar}\right)$ as a function of the planetary radiative equilibrium temperature, $T_{\text {eq }}$. Results for an assumed magnetic field strength $B_{\text {dip }}=3,10$ and $30 \mathrm{G}$ are shown as solid, dashed and dash-dotted lines, respectively.

These curves cover three different regimes for momentum balance in the thermallyforced atmospheres of hot giant exoplanets. At low $T_{\text {eq }}$ values, advection reduces the daynight temperature differential $\Delta T_{\text {horiz }}$ according to Eq. ([6), which leads to the relatively steep slope of $V_{\phi}$ with $T_{\text {eq }}$. As shown best by the solid line, at higher $T_{\text {eq }}$ values, this slope becomes shallower as the atmosphere becomes radiatively dominated (when $\tau_{\text {adv }} / \tau_{\text {rad }}>1$ ), with a negligible role for advection (as defined by Eq. [6]). Finally, the three curves show that magnetic drag eventually becomes efficient at braking the winds and that it does so at lower radiative equilibrium temperatures for stronger magnetic field strengths. Results comparable to those shown in Fig. 1 are obtained for deeper levels in the weather layer than the thermal photosphere.

The exponential decline in $V_{\phi}$ at high $T_{\text {eq }}$ values, which is caused by strong magnetic drag, can be understood as follows. As resistivity drops, the dominant balance in the momentum equation (Eq. [5]) eventually becomes one where the pressure gradient acceleration term is balanced by the deceleration term from the bulk Lorentz force. In that limit, $V_{\phi}$ becomes small and simple dimensional analysis in that limit shows that it scales in proportion to the resistivity, $V_{\phi} \propto \eta$, which itself is an exponentially decreasing function of the atmospheric temperatures in the weather layer, and thus of $T_{\text {eq }}$. 


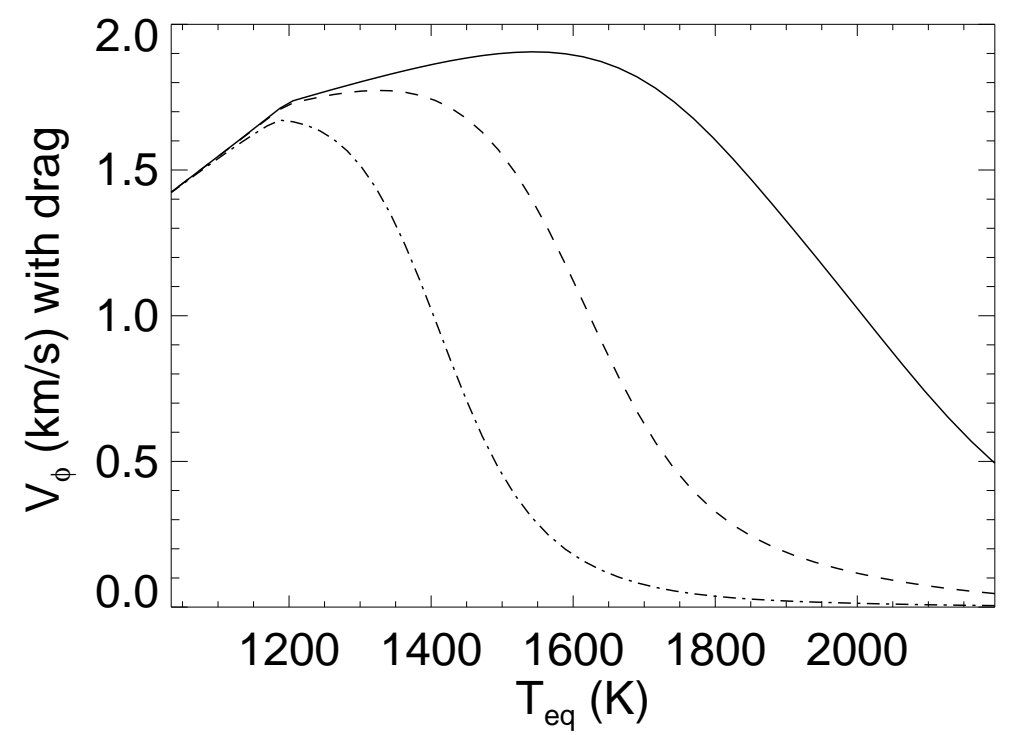

Fig. 1. - Estimate of the zonal wind speed, $V_{\phi}($ in $\mathrm{km} / \mathrm{s})$, at the thermal photosphere as a function of the planetary radiative equilibrium temperature, $T_{\text {eq }}$ (in $K$ ). Solid, dashed and dash-dotted lines show results for surface magnetic field strengths $B_{\text {dip }}=3,10$ and $30 \mathrm{G}$, respectively. The exponential decline of $V_{\phi}$ at high $T_{\text {eq }}$ is caused by strong magnetic drag. 


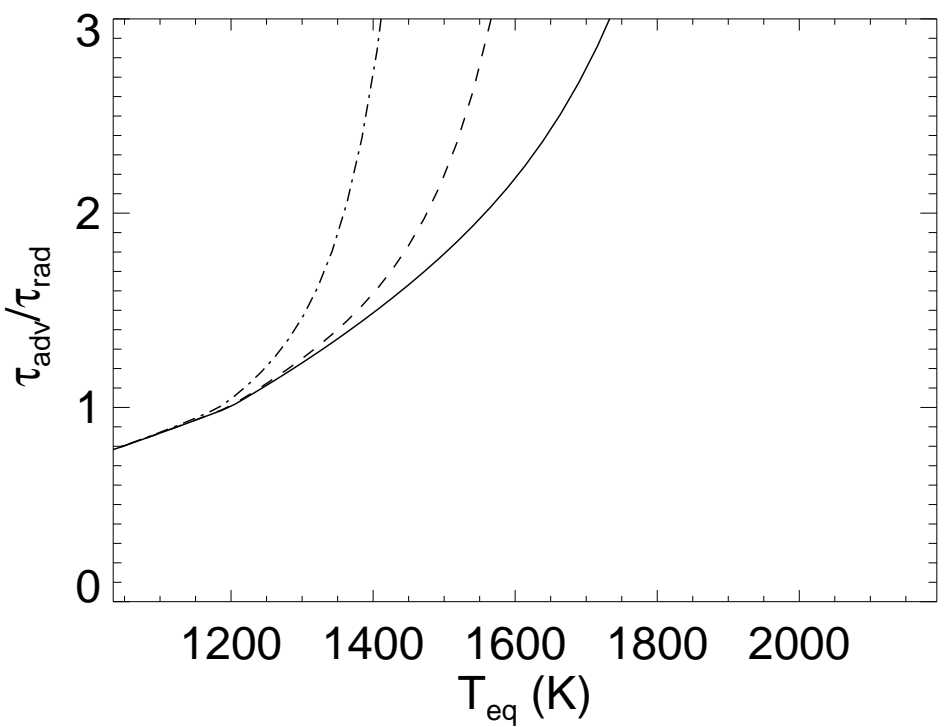

Fig. 2.- Ratio of advective to radiative timescales, $\tau_{\text {adv }} / \tau_{\text {rad }}$, at the thermal photosphere as a function of the planetary radiative equilibrium temperature, $T_{\text {eq }}$ (in K). Solid, dashed and dash-dotted lines show results for surface magnetic field strengths $B_{\text {dip }}=3,10$ and $30 \mathrm{G}$, respectively. The radiative equilibrium temperature above which advection becomes negligible $\left(\tau_{\text {adv }} / \tau_{\text {rad }}>1\right)$ is comparable to the temperature at which the strong magnetic drag regime begins for $B_{\text {dip }} \sim 30 \mathrm{G}$. 


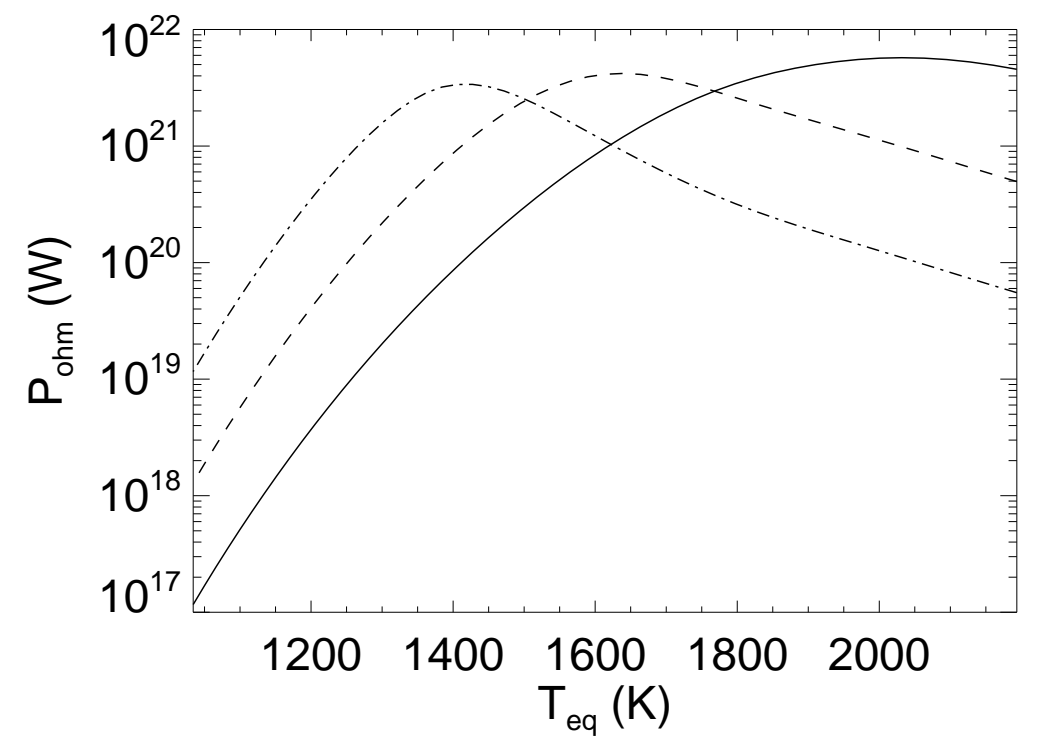

Fig. 3.- Ohmic power (in Watts) dissipated as a result of induction in the weather layer as a function of the planetary radiative equilibrium temperature, $T_{\text {eq }}$ (in $\mathrm{K}$ ). Solid, dashed and dash-dotted lines show results for surface magnetic field strengths $B_{\text {dip }}=3,10$ and $30 \mathrm{G}$, respectively. Ohmic power rises exponentially with $T_{\text {eq }}$ until the regime of strong magnetic drag is reached, at which point the power starts declining exponentially. 


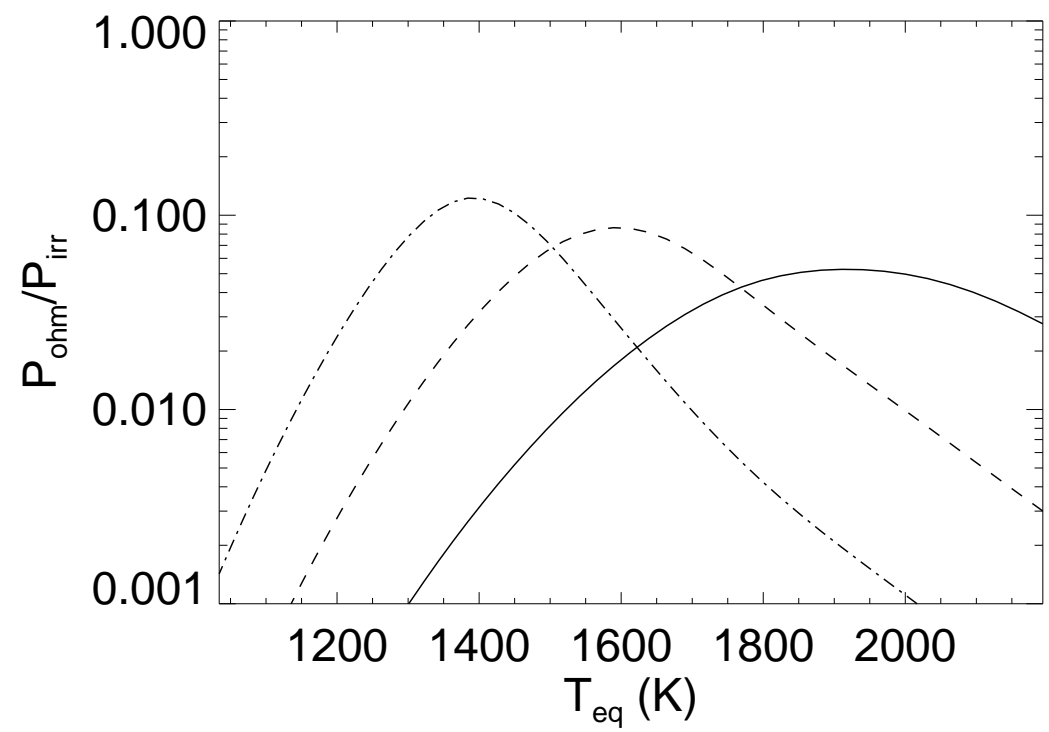

Fig. 4.- Ratio of ohmic to irradiation power $\left(P_{\mathrm{ohm}} / P_{\mathrm{irr}}\right)$ as a function of the planetary radiative equilibrium temperature, $T_{\text {eq }}$ (in $\mathrm{K}$ ). Solid, dashed and dash-dotted curves show results for surface magnetic field strengths $B_{\text {dip }}=3,10$ and $30 \mathrm{G}$, respectively. Assuming that a ratio $P_{\text {ohm }} / P_{\text {irr }}>1 \%$ is required for sizable planetary radius inflation, this figure illustrates how radius inflation should only exist over a restricted range of equilibrium temperatures, according to our magnetic scaling laws. 
Figure 2 illustrates the nature of the change from an advective to a radiative behavior for hot giant exoplanet atmospheres as the radiative equilibrium temperature, $T_{\text {eq }}$, is raised above $\sim 1200 \mathrm{~K}$. Below $1200 \mathrm{~K}, \tau_{\text {adv }} / \tau_{\text {rad }}<1$ and advection reduces the day-night temperature differential according to Eq. (6) . Above $1200 \mathrm{~K}$, however, the atmospheric thermal structure (at the thermal photosphere) becomes essentially radiative, with little role, if any, for advection. As shown by the three curves in Fig. 2, this transition is effectively accelerated by magnetic drag, when it significantly reduces the wind speeds, the more so for strong magnetic field strengths.

Figure 3 shows the ohmic power $P_{\text {ohm }}$ dissipated as a result of induction in the weather layer as a function of the planetary radiative equilibrium temperature, $T_{\text {eq. }}$. The exponential rise and subsequent exponential decline of $P_{\text {ohm }}$ with $T_{\text {eq }}$ can be understood as follows. In the limit of weak magnetic drag, $V_{\phi}$ is independent of the resistivity and it varies only weakly with $T_{\text {eq }}$. Equation (9) then implies that $P_{\mathrm{ohm}} \propto Q_{\mathrm{ohm}} \propto 1 / \eta$. On the other hand, in the limit of strong magnetic drag, since $V_{\phi} \propto \eta$, the ohmic power declines exponentially with $T_{\text {eq }}$ according to $P_{\mathrm{ohm}} \propto Q_{\mathrm{ohm}} \propto \eta$. As shown by Fig. 3, the radiative equilibrium temperature at which the ohmic power peaks depends sensitively on the value of the surface magnetic field strength.

Figure 4 shows a different representation of the ohmic dissipation results, where the ohmic power $P_{\text {ohm }}$ has been scaled to the total irradiation power $P_{\text {irr }}=4 \pi R_{P}^{2} \sigma T_{\text {eq }}^{4}$ received by a hot giant exoplanet with radiative equilibrium temperature, $T_{\text {eq }}$. The ratio $P_{\text {ohm }} / P_{\text {irr }}$ is useful because it has been suggested that a value $\gtrsim 1 \%$ is needed for sizable radius inflation of a hot Jupiter 3 by deposition either in the bulk convective interior (Batygin \& Stevenson 2010) or in the deep atmosphere (Perna et al. 2010b). Our simple magnetic scaling laws lack the descriptive power to identify where in the planet the ohmic power is dissipated, but they clarify the range of radiative equilibrium temperatures over which such power is in principle available for radius inflation. Like the peak ohmic value, the range of radiative equilibrium temperatures over which $P_{\mathrm{ohm}} / P_{\text {irr }}>1 \%$ (or any other fixed ratio) is a sensitive function of the surface magnetic field strength.

\footnotetext{
${ }^{3}$ We note that any energetic threshold for radius inflation, such as the ratio $P_{\text {ohm }} / P_{\text {irr }}$, will generally be a function of the planet's mass and age (e.g., Miller et al. 2009), so that the $1 \%$ value adopted here should be taken as merely indicative.
} 


\section{Discussion}

\subsection{Parameter Dependencies}

An examination of the various assumptions made in deriving our magnetic scaling laws makes it clear that they can only capture the most basic, order-of-magnitude behavior for hot giant exoplanet atmospheres across the wide range of radiative equilibrium temperatures considered. It is important for the validity of the scaling laws that the central physical ingredient of the theory, atmospheric resistivity, varies exponentially with atmospheric temperatures, via thermal ionization balance. Still, there is some freedom in renormalizing the scaling laws by adopting different parameter values than the fiducial ones we have adopted. It is thus important to understand the sensitivity of the scaling law results to variations in the different model parameters.

We find that modest changes $(\sim 20 \%)$ in the assumed values of the planetary radius, $R_{p}$, the gravitational acceleration, $g$, the perfect gas constant, $\mathcal{R}$, or the specific heat at constant pressure, $C_{p}$, have only minor quantitative effects on the scaling law results. Raising the assumed gas metallicity to ten times solar, which increases the free electron density accordingly, reduces the temperature at which the strong drag regime is entered (from, e.g., $\sim 1600 \mathrm{~K}$ to $\sim 1475 \mathrm{~K}$, for $B_{\text {dip }}=10 \mathrm{G}$ ), but the scaling law results are otherwise qualitatively similar to the solar metallicity case. Similarly, changing the power law index used to evaluate the day-night temperature differential in Eq. (6) from $n=1$ to $n=3$ or $n=5$ further steepens the slope of $V_{\phi}$ with $T_{\text {eq }}$ at low $T_{\text {eq }}$ values (leftmost region in Fig. (1), but the scaling law results remain otherwise qualitatively similar.

The scaling law results are sensitive to variations in model parameters which directly affect the atmospheric temperatures in the weather layer, however, as expected from the exponential dependence of the resistivity with temperature. For example, increasing the thermal opacity coefficient, $k_{\mathrm{th}}$, by $50 \%$ nearly doubles the ohmic power and shifts the ohmic peak from $\sim 1600 \mathrm{~K}$ to $\sim 1500 \mathrm{~K}$, for $B_{\text {dip }}=10 \mathrm{G}$. Reducing $k_{\text {th }}$ by a factor of two leads to about three times less ohmic dissipation, a peak ohmic power at $\sim 1650 \mathrm{~K}$ for $B_{\text {dip }}=10 \mathrm{G}$ and magnetic Reynolds numbers reduced by a factor of a few. Increasing the visible opacity coefficient, $k_{\mathrm{v}}$, by $50 \%$ reduces the ohmic power by a factor $\sim 5$, shifts the ohmic peak to slightly lower temperatures and reduces magnetic Reynolds numbers by a factor of a few. Reducing the value of $k_{\mathrm{v}}$ by a factor of two increases the ohmic power by a factor $\sim 5$, shifts the ohmic peak to slightly larger temperatures and increases magnetic Reynolds numbers by a factor of a few.

Similarly, an increase in the assumed dayside dilution factor, $f$, entering Guillot's radiative solution raises the ohmic power somewhat and shifts its peak to lower temperature 
(from, e.g, $\sim 1600 \mathrm{~K}$ for $f=0.5$ to $\sim 1475 \mathrm{~K}$ for $f=0.7$, for $B_{\text {dip }}=10 \mathrm{G}$ ). A reduction in the magnitude of the maximum day-night temperature differential in Eq. (6) from $0.5 T_{\text {day }}$ to $0.3 T_{\text {day }}$ reduces the ohmic power by about a factor two and the peak $V_{\phi}$ values by $\sim 25 \%$. Conversely, an increase of this maximum day-night temperature differential from $0.5 T_{\text {day }}$ to $0.7 T_{\text {day }}$ doubles the ohmic power and increases the peak $V_{\phi}$ value by $\sim 20 \%$.

Another factor which may significantly impact atmospheric temperatures in the weather layer, and thus the magnetic scaling laws, is the possibility of a temperature inversion in the upper atmosphere of strongly-irradiated giant exoplanets (e.g., Fortney et al. 2008). Hot giant exoplanets with such inversions would typically have reduced temperatures in their weather layer, from extra absorption of stellar light at altitude. Even though we did not explore this scenario in any detail, and instead adopted a simple set of Guillot (2010) radiative solutions with $\kappa_{\mathrm{th}}>\kappa_{\mathrm{v}}$ for our thermal profiles, one can anticipate a systematic shift of the magnetic scaling law results at fixed $T_{\text {eq }}$ for those planets with inversions, in proportion to the temperature deficit in their weather layer. A detailed investigation of the effects of temperature inversions on magnetic scaling laws would constitute a valuable extension of our work.

Besides the strong dependence on the planetary magnetic field strength, which is illustrated explicitly in Figs. 1-4, our parameter space exploration reveals a strong dependence of the scaling law results on parameters affecting the atmospheric temperatures in the modeled weather layer. This suggests that improved treatments of the atmospheric thermal structure, e.g. based on three-dimensional modeling, would greatly benefit efforts to better understand the magnetic behavior of hot giant exoplanet atmospheres as a function of their radiative equilibrium temperature.

\subsection{Magnetic Reynolds Number and Induced Fields}

As the radiative equilibrium temperature of a hot giant exoplanet is raised, the degree of coupling between the atmospheric flow and the planetary magnetic field also increases. The nature of this coupling in the strong drag limit identified by our scaling laws, at the high end of the range of equilibrium temperatures considered, is an important issue.

Figure 5 shows an estimate of the magnetic Reynolds number of the atmospheric flow, $R_{m}$, as a function of the planetary radiative equilibrium temperature, $T_{\text {eq }}$, using the same notation as in previous figures. This magnetic Reynolds number is evaluated as

$$
R_{m}=\frac{V_{\phi} H_{p}}{\eta}
$$


where $V_{\phi}$ is the zonal wind velocity at the thermal photosphere (Fig. 1), $H_{p}$ is the pressure scale height and $\eta$ is the resistivity. Fig. 5 reveals that the magnetic Reynolds number is less than unity and independent of the magnetic field strength at low enough radiative equilibrium temperatures. Beyond $T_{\text {eq }} \sim 1300 \mathrm{~K}$, however, $R_{m}$ exceeds unity and reaches different, near-asymptotic values for different strengths of the planetary magnetic field. The near-asymptotic behavior of $R_{m}$ at high temperatures can easily be understood as resulting from the previously established scaling $V_{\phi} \propto \eta$ in the strong drag limit, with different normalizations for the different magnetic field strengths.

Traditionally, the regime $R_{m} \gtrsim 1$ would indicate a significant degree of coupling between the flow and the magnetic field, and the possibility of sustained dynamo action in the atmosphere. It is unclear, however, whether the moderately large values of $R_{m}$ found in Fig. 1 are sufficient to trigger a self-sustained dynamo, and what the nature of such a dynamo might be, given the unusual setup of a very shallow atmospheric flow threaded by the planetary magnetic field.

Irrespective of the issue of sustained dynamo action, the significant gas-field coupling arising from values of $R_{m} \gtrsim 1$ raises the possibility of large induced field strengths even in the simple kinematic framework adopted in this study. In particular, a strong enough induced toroidal magnetic field $\left(B_{\phi}\right)$ could in principle result in secondary induction of a strong poloidal field component by meridional atmospheric motions. This could challenge one of our basic assumption, which is that a dominant contribution to the poloidal field component is provided by the planetary dipole field ( $B_{\text {dip }}$ in Eq. [1]).

Some insight on this issue may be gained by simple dimensional analysis. Using the same scaling as in $\$ 3.4$ for the latitudinal current, $J_{\theta} \sim c \mathrm{~V}_{\phi} B_{\text {dip }} /(4 \pi \eta)$, and assuming that the relevant gradient scale is $\sim H_{p}$ in the relation between the induced current and the induced field, $J_{\theta}=(c / 4 \pi) \nabla \times B_{\phi}$, one deduces a simple estimate of the strength of the induced toroidal field in terms of the background dipole field: $B_{\phi} \sim R_{m} B_{\text {dip }}$. In the strong drag limit, this suggests values for the induced toroidal field which can be in excess of the planetary dipole field.

If large enough field values were induced into a poloidal component by meridional motions, Eq. (1), which is a starting point for our scaling laws, could cease to be valid: the value of the poloidal field may no longer be set by the fixed value of the planetary dipole field but rather by multi-dimensional induction in the atmospheric flow itself. It is unclear, however, whether such large induction of poloidal field is expected in the weather layer of hot giant exoplanets. Indeed, simple dimensional analysis analogous to the one performed above suggests an induced poloidal field strength $B_{\text {pol }} \sim R_{m}^{*} B_{\phi}$, where $R_{m}^{*}=V_{\theta} H_{p} / \eta$ is the magnetic Reynolds number associated with the meridional (rather than zonal) flow. Therefore, to the 


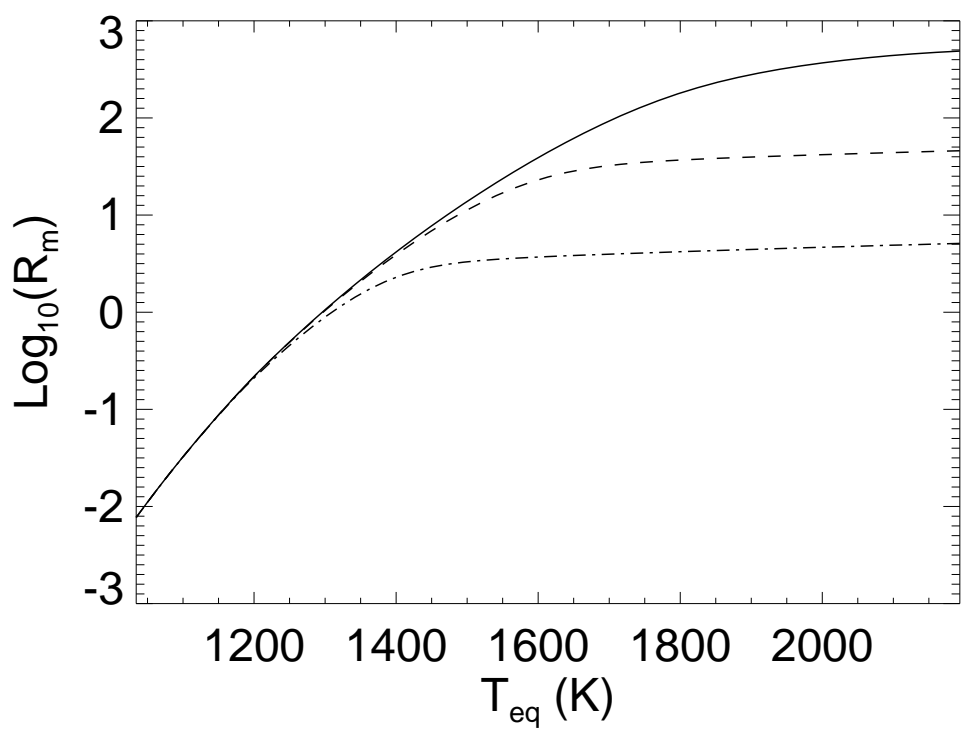

Fig. 5.- Logarithm of the magnetic Reynolds number, $R_{\mathrm{m}}$, of the atmospheric flow at the thermal photosphere, as a function of the planetary radiative equilibrium temperature, $T_{\mathrm{eq}}$ (in K). Solid, dashed and dash-dotted lines show results for a surface magnetic field strength $B_{\text {dip }}=3,10$ and $30 \mathrm{G}$, respectively. The exponential drop in $V_{\phi}$ caused by strong magnetic drag is responsible for the near-asymptotic values of $R_{\mathrm{m}}$ reached at high $T_{\text {eq }}$. 
extent that the meridional flow velocities $\left(V_{\theta}\right)$ are much weaker than the zonal ones, in the presence of separate drags acting on each component, $R_{m}^{*}$ may still be small enough for the induced poloidal field to remain negligible compared to the background dipole field.

Our scaling law results in the strong drag limit assume that the planetary dipole field remains dominant relative to any induced poloidal field. It is difficult to evaluate if or when this assumption might break down without considerably more detailed explorations of the physics of magnetized atmospheric flows than performed here, such as multi-dimensional models with distinct drag treatments for the zonal and meridional flows, or perhaps even full MHD treatments to explore dynamo issues. Until such studies become available, it will thus be important to remain cautious when using simple scaling arguments like ours to interpret the phenomenology of hot giant exoplanet atmospheres in the strong drag limit.

\subsection{Connection to Previous Work}

Our scaling law results are broadly consistent with previously published results for the zonal wind speeds in the weather layer of hot giant exoplanets. In particular, the values shown in Fig. 1 for zonal wind speeds are qualitatively consistent with the zonallyaveraged results of atmospheric circulation models for the hot giant exoplanet HD209458b $\left(T_{\text {eq }} \sim 1400 \mathrm{~K}\right)$ with weak $\left(B_{\text {dip }}=3 \mathrm{G}\right)$, moderate $\left(B_{\text {dip }}=10 \mathrm{G}\right)$ and strong $\left(B_{\text {dip }}=30 \mathrm{G}\right)$ imposed drag, as reported in Perna et al. (2010a). Extrapolating the trend at low $T_{\text {eq }}$ values in Fig. 1 also points to zonal wind speeds in broad agreement with those reported by Lewis et al. (2010) for the atmosphere of the hot Neptune GJ436b $\left(T_{\text {eq }} \sim 650 \mathrm{~K}\right)$ and it supports the notion that the atmospheric flow on this cooler exoplanet can effectively be treated as free of magnetic drag.

The nature of the transition from the unmagnetized to the magnetized atmosphere regime for hot giant exoplanets is a particularly interesting issue to examine in light of our scaling law results. In terms of magnetic drag, Fig. 1 indicates that the magnetized regime starts around $T_{\text {eq }} \sim 1200-1600 \mathrm{~K}$, depending on the strength of the planetary magnetic field. Defining the value of $T_{\text {eq }}$ above which ohmic dissipation begins to be important is more difficult because it depends on a threshold value for the ratio of ohmic to irradiation power above which significant radius inflation is expected. This ratio is not well defined because it depends on details of the ohmic dissipation process but a threshold value of $P_{\text {ohm }} / P_{\text {irr }} \sim 1 \%$ is probably a reasonable guess (Guillot \& Showman 2002; Batygin \& Stevenson 2010; Perna et al. 2010b). As shown in Fig. 4, this $1 \%$ threshold is reached at $T_{\text {eq }} \sim 1100-1500 \mathrm{~K}$ according to our scaling laws, with a strong dependence on the planetary magnetic field strength. When evaluating the transition in the magnetized regime, one should remember 
the great degree of simplification used in deriving our scaling laws, which is exemplified by some of the strong parameter dependencies discussed in 95.1 . The nature of the spatial distribution of ohmic dissipation will also likely influence the transition temperature for the onset of radius inflation (Batygin \& Stevenson 2010; Perna et al. 2010b).

In their study of the heavy element content of giant exoplanets, Miller \& Fortney (2011) suggest a temperature of $\sim 1000 \mathrm{~K}$ as the threshold below which radius inflation appears to be absent from their sample of exoplanets. This temperature threshold is broadly consistent with, but somewhat lower than, the threshold values inferred on the basis of our scaling law results, shown in Fig. 4. It may be possible to bring these threshold values into closer agreement by lowering the critical value of $P_{\mathrm{ohm}} / P_{\text {irr }}$ required for sizable radius inflation (below our $1 \%$ assumption) and/or by postulating that planetary magnetic fields in the exoplanet sample considered by Miller \& Fortney (2011) are generally strong ( $\left.B_{\text {dip }} \gtrsim 30 \mathrm{G}\right)$.

In an analysis sharing some similarities with the present one, Laughlin et al. (2011) have studied a correlation between a measure of radius inflation for hot giant exoplanets, the so-called radius anomaly, and their effective temperature 4 in an observationally constrained sample. The emergence of a positive radius anomaly at $T_{\text {eff }} \gtrsim 1000 \mathrm{~K}$, as shown in their Fig. 2, is broadly consistent with our scaling law results. It is unclear whether or not our work supports the power law dependence of the radius anomaly with $T_{\text {eff }}$ advocated by Laughlin et al. (2011). This relation depends on the intricate physics that connects ohmic dissipation with the effective radius inflation and it simply is not addressed by our scaling laws. It is conceivable, however, that the large spread in the amount of radius anomaly found at any $T_{\text {eff }}$ value (see their Fig. 2) could be accounted for by variations in the strengths of the planetary magnetic field in the sample, since we find $B_{\text {dip }}$ to be a rather sensitive parameter of the theory. We note that there also is an interesting indication of a systematic drop in radius anomaly at $T_{\text {eff }} \gtrsim 1900 \mathrm{~K}$ in the Fig. 2 of Laughlin et al. (2011). It is possible that this feature is associated with the drop in ohmic dissipation, and thus in the amount of radius inflation, that is expected in the strong drag regime according to our scaling law results (see our Fig. 41).

Batygin et al. (2011) have studied in detail some of the consequences of ohmic dissipation for the thermal evolution of hot giant exoplanets. In their analysis, these authors assume that the global efficiency, $\epsilon$, of ohmic heating relative to that from stellar irradiation is a model constant, fixed at values of 1,3 or $5 \%$. Our scaling law results challenge this simplifying assumption since we find that the ratio $P_{\mathrm{ohm}} / P_{\mathrm{irr}}$ is a strong function of both

\footnotetext{
${ }^{4}$ For strongly-irradiated planets with negligible internal heat fluxes, effective and radiative equilibrium temperatures are equivalent.
} 
the planetary effective temperature, $T_{\text {eff }}$, and the planetary magnetic field strength (see our Fig. 4). The emergence of sizable radius inflation at $T_{\text {eff }} \gtrsim 1400 \mathrm{~K}$ in the evolutionary models of Batygin et al. (2011; see their Figs 6-8) is certainly consistent with the typical threshold values suggested by our scaling laws, but the existence of a well-defined transition temperature would require a rather uniform set of planetary magnetic strengths according to our analysis. Given the difference in methodology, we note that it may be possible to recast some of the results of Batygin et al. (2011) in the language of our scaling laws, by considering that their assumed ohmic efficiency values of $\epsilon=1,3$ and $5 \%$ correspond to different effective planetary magnetic field strengths.

Batygin et al. (2011) argued that magnetic drag has a very limited effect on the magnitude of zonal winds in hot giant exoplanet atmospheres. This is inconsistent with our scaling law results, which do suggest the existence of a strong drag regime, as shown in our Fig. 1, and it also conflicts with the strongly dragged simulation results described in Perna et al. (2010b) and Rauscher \& Menou (2011). Our work suggests that the emergence of a strong drag regime is essential to the saturation and the eventual decline of ohmic dissipation at high planetary effective temperatures. It is worth noting that a systematic reduction in the efficiency of ohmic dissipation $\epsilon$ in the models of Batygin et al. (2011) when $T_{\text {eff }} \gtrsim 1600-$ $1900 \mathrm{~K}$ would likely bring their model radii in closer agreement with the data (see their Figs. 6-8). Such a drop of $\epsilon$ with $T_{\text {eff }}$ could also mitigate the emergence of unstable, overflowing evolutionary tracks identified by Batygin et al. (2011) for models with high enough values of $T_{\text {eff }}(\gtrsim 1400-1800 \mathrm{~K})$.

\subsection{Observational Signatures}

Magnetic drag and ohmic dissipation are separate manifestations of the same magnetic induction process operating in the atmosphere of a hot giant exoplanet. It is of great interest to explore the type of observational signatures expected from magnetic drag and ohmic dissipation since these observables should ultimately be related to each other. As discussed earlier, the most direct observable manifestation of ohmic dissipation is planetary radius inflation. Signatures of magnetic drag, on the other hand, would most likely emerge in the form of reduced atmospheric wind speeds.

Over the past few years, it has become possible to constrain the magnitude of wind advection in the atmosphere of a few hot giant exoplanets, by measuring their infrared phase curves (e.g., Harrington et al. 2006; Cowan et al. 2007; Knutson et al. 2007, 2009a,b; Crossfield et al. 2010). The standard interpretation that has been given to an infrared peak emission that is offset from the planet's orbital phase is that heat is advected away from the 
dayside by eastward equatorial winds at the planet's thermal photosphere (e.g., Showman et al. 2008, 2009, 2010; Rauscher \& Menou 2010; Dobbs-Dixon et al. 2010; Burrows et al. 2010; Heng et al. 2011; Showman \& Polvani 2011). Magnetic drag will act to reduce the speed of zonal winds and thus the amount of heat advection away from the dayside, so that atmospheres experiencing stronger magnetic drag may exhibit reduced infrared phase offsets (see, e.g., Fig. 3 of Rauscher \& Menou 2011)

A simple measure of the degree of heat advection and infrared phase offset is provided by the ratio $\tau_{\text {adv }} / \tau_{\text {rad }}$ shown in Fig. 2. As $T_{\text {eq }}$ increases, $\tau_{\text {rad }}$ decreases steeply (see Eq. [8]) but ultimately it is the exponential decline experienced by the zonal wind speed $V_{\phi}$ in the strong drag limit that leads to the large values of $\tau_{\text {adv }} / \tau_{\text {rad }}$ at high temperatures in Fig. 2, One would thus expect smaller infrared phase offsets for giant exoplanets with higher radiative equilibrium temperatures. It is difficult to evaluate the magnitude of such offsets with the simple ratio $\tau_{\text {adv }} / \tau_{\text {rad }}$, as opposed to detailed atmospheric circulation models, but we note that HD189733b, with $T_{\text {eq }} \simeq 1200 \mathrm{~K}$, still has a measurable offset (Knutson et al. 2007, 2009a) for a value of $\tau_{\text {adv }} / \tau_{\text {rad }} \sim 1$ (according to Fig. 2, see also discussion in Agol et al. 2010).

Interestingly, our scaling law results indicate that the radiative equilibrium temperature at which ohmic dissipation peaks is comparable to that at which magnetic drag reduces the zonal wind speed by about a half (relative to the case without magnetic drag). A comparison between Figures 1 and 4 reveals that this trend holds independently of the planetary magnetic field strength. It suggests that, across a range of $T_{\text {eq }}$ values, from the start of the magnetized regime until the ohmic peak is reached (e.g., $\sim 1300-1600 \mathrm{~K}$ for $B_{\text {dip }}=10 \mathrm{G}$ ), zonal wind speeds will systematically drop as ohmic dissipation increases. Observationally, this might translate into an anticorrelation between the magnitude of infrared phase offsets and the degree of radius inflation of hot giant exoplanets. At higher temperatures, beyond the ohmic peak, one would expect both zonal wind speeds and ohmic dissipation to drop with $T_{\text {eq }}$. The large values of the ratio $\tau_{\text {adv }} / \tau_{\text {rad }}(\gg 1)$ expected at such high temperatures may prevent any observable infrared phase offset, but the decline in ohmic dissipation may result in a gradual disappearance of the radius inflation phenomenon with $T_{\text {eq }}$, as already mentioned above in connection to Fig. 2 of Laughlin et al. (2011). Cowan \& Agol (2011) have also noticed that hot Jupiters receiving the largest irradiation fluxes (corresponding to $T_{\text {eq }} \gtrsim 1900 \mathrm{~K}$ ) appear to be inefficient at redistributing heat to their night sides, a trend which may be consistent with the emergence of a strong drag regime. Given the idealized nature of our scaling laws, it will be interesting to put these various arguments to the observational test and to interpret the results with more detailed, possibly planet-specific atmospheric models. 


\section{Conclusion}

We developed scaling laws addressing the magnitude of magnetic drag and ohmic dissipation in the atmospheres of hot giant exoplanets, as a function of the planetary radiative equilibrium temperature. These scaling laws shed light on the nature of the transition from an unmagnetized to a magnetized regime of atmospheric behavior, at $T_{\text {eq }} \gtrsim 1000 \mathrm{~K}$, and on the emergence of a strong drag regime at even higher temperatures. Assuming that poloidal field induction remains weak relative to toroidal induction, an issue which deserves further scrutiny, our scaling laws suggest that ohmic dissipation eventually vanishes in the limit of very strong drag. Clarifying the role of magnetic drag and ohmic dissipation for hot giant

exoplanets is important and we have suggested that their interplay could manifest in the form of an anticorrelation between the amount of infrared phase offset and the degree of radius inflation for such planets, thus linking the atmospheric and interior properties of hot giant exoplanets in an observationally testable way.

The author thanks Charles Gammie for providing the original ionization balance routine used in this work. This work was supported in part by NASA grants JPLCIT 1366188 and PATM NNX11AD65G.

\section{REFERENCES}

Agol E. et al. 2010, ApJ 721, 1861

Baraffe, I., Chabrier, G., Barman, T. 2010, Reports on Progress in Physics, Vol. 73, Issue 1, pp. 016901

Batygin, K. \& Stevenson, D. J. 2010, 714, L238

Batygin, K., Stevenson, D. J. \& Bodenheimer, P. H. 2011, ApJ submitted, arXiv:1101:3800

Burrows, A., Rauscher, E., Spiegel, D. S. \& Menou, K. 2010, ApJ 719, 341

Burrows, A. \& Orton, G. 2010, in "Exoplanets", Space Science Series of the University of Arizona Press (Tucson, AZ); Ed. S. Seager; arXiv:0910.0248

Charbonneau, D. 2009, in "Transiting Planets", Proceedings of the International Astronomical Union, IAU Symposium, Volume 253, p. 1-8

Cowan, N. B., Agol, E. \& Charbonneau, D. 2007, MNRAS 379, 641

Cowan, N. B. \& Agol, E. 2011 ApJ 729, 54

Crossfield, I. et al. 2010, ApJ 723, 1436 
Dobbs-Dixon, I., Cumming, A., Lin, D. N. C. 2010, ApJ, 710, 1395

Fortney, J. J., Lodders, K., Marley, M. S. \& Freedman, R. S. 2008, ApJ 678, 1419

Goody, R. M. \& Yung, Y. L. 1989, Atmospheric radiation : theoretical basis. 2nd ed, New York, NY: Oxford University Press

Guillot, T. 2010, A\&A 520, A27

Guillot, T. \& Showman, A. P. 2002, A\&A 385, 156

Hansen, B. M. S. 2008, ApJS 179, 484

Harrington, J. et al. 2006, Science 314, 623

Heng, K., Menou, K. \& Phillipps, P. J. 2011, MNRAS 413, 2380

Knutson, H. A. et al., 2007, Nature 447, 183

Knutson, H. A. et al., 2009a, ApJ 690, 822

Knutson, H. A. et al., 2009b, ApJ 703, 769

Laughlin, G., Crismani, M. \& Adams, F. C. 2011, ApJ 729, L7

Lewis, N. K. et al. 2010, ApJ 720, 344

Liu, J., Goldreich, P. M., Stevenson, D. J. 2008, Icar., 653, 664

Miller, N. \& Fortney, J., 2011 ApJ 736, L29

Miller, N., Fortney, J. \& Jackson, B., 2009 ApJ 702, 1413

Perna, R., Menou, K. \& Rauscher, E., 2010a, ApJ 719, 1421

Perna, R., Menou, K. \& Rauscher, E., 2010b, ApJ 724, 313

Rauscher, E. Menou, K. 2010, ApJ 714, 1334

Rauscher, E. Menou, K. 2011, ApJ submitted, arXiv:1105.2321

Sato, M. 1991, Contrib. Plasma Phys. 31(3), 331

Showman, A. P. et al. 2009, ApJ 699, 564

Showman, A. P., Y-K. Cho, J., Menou, K. 2010, in "Exoplanets", Space Science Series of the University of Arizona Press (Tucson, AZ); Ed. S. Seager; arXiv:0911.3170.

Showman, A. P., Menou, K., Cho, J. Y.-K. 2008, in "Extreme Solar Systems", ASP Conference Series, Vol. 398, proceedings of the conference held 25-29 June, 2007, at Santorini Island, Greece. Edited by D. Fischer, F. A. Rasio, S. E. Thorsett, and A. Wolszczan, p.419; arXiv:0710.2930

Showman, A. P. \& Polvani, L. M. 2011, ApJ 738, 71 
Winn, J. 2010, in "Exoplanets", Space Science Series of the University of Arizona Press (Tucson, AZ); Ed. S. Seager; arXiv:1001.2010

Zhu, X., Talaat, E. R., Baker, J. B. H. \& Yee, H.-H. 2005, Ann. Geo. 2005, 23, 3313 